\title{
Conceptualización y evaluación de las competencias para el análisis, reflexión y semiosis didáctica. El caso de los estudiantes para profesor de matemáticas ${ }^{1}$
}

\author{
Conceptualization and assessment of competencies for analysis, reflection \\ and didactic semiosis. The case of mathematics student teachers \\ Conceituação e avaliação de competências para a análise, reflexão e \\ didática semiose. 0 caso dos futuros professores de matemática
}

Jorge Orlando Lurduy Ortegón ${ }^{2}$

\begin{abstract}
Resumen
En este artículo se presentan los resultados de la investigación doctoral "Conceptualización y evaluación de las competencias para el análisis, reflexión y semiosis didáctica. El caso de los estudiantes para profesor de matemáticas". La investigación tiene un carácter propositivo de tipo teórico-metodológico, aborda el problema de la caracterización y la evaluación de la competencia del profesor de matemáticas para la implementación de procesos de estudio matemáticos en el aula. Desde las perspectivas de la teoría de la complejidad, del enfoque ontosemiótico del conocimiento y la instrucción matemática y la incorporación sistemática de herramientas de la semiótica de Peirce, se articulan planteamientos propios de los enfoques metodológicos que desarrollan teoríasa partir de los datos, el análisis cualitativo del contenido y el análisis semiótico de textos. Las dimensiones, categorías e indicadores de la competencia identificada y evaluada están apoyados en los datos textuales proporcionados por profesores en su formación inicial.
\end{abstract}

Palabras clave: evaluación, competencia, análisis, reflexión, semiosis, didáctica, complejidad, semiótica.

\begin{abstract}
This paper presents doctoral research results "Conceptualization and assessment of competencies for analysis, reflection and didactic semiosis. The case of the initial training of teacher mathematics". Research has a proactive nature of a theoretical-methodological in addressing the problem of the characterization and assessment of competence math teacher to implement mathematical study processes in the classroom. From the perspective of complexity theory, knowledge ontosemiotic approach and mathematics instruction and mainstreaming tools Peirce's semiotics, owned approaches articulated methodological approaches that develop theory from the data, the analysis qualitative content and semiotic
\end{abstract}

1 Artículo de investigación. Este artículo es una síntesis de los procesos teórico-metodológicos realizados y del informe final de la investigación doctoral que fue dirigidapor el profesor Juan D. Godino

2 Profesor de la licenciatura en Educación Básica con Énfasis en Matemáticas de La Universidad Distrital Francisco José De Caldas. Bogotá- Colombia. Contacto: jolurduy@udistrital.edu.co 


\section{Introducción}

Los aspectos tomados en cuenta con la temática de esta investigación conciernen, en general, a la educación matemática, a la didáctica de las matemáticas y a su investigación, y están relacionados con el estudio de los programas de formación inicial de profesores de matemáticas.De forma focalizada, se investiga la temática del desarrollo profesional del profesor de matemáticas al abordar el tema de las competencias de los profesores de matemáticas; específicamente, se evalúan las competencias de los estudiantes para profesor de matemáticas (EPM), desarrolladas en su proceso formativo como docentes.

Esta investigación se desarrolló en el programa de formación de profesores de matemáticas de la Licenciatura en Educación Básica con Énfasis en Matemáticas (LEBEM) de la Universidad Distrital Francisco José de Caldas de Bogotá, Colombia. Focalmente este estudio se interesa por indagar las acciones didácticas de los estudiantes para profesor de matemáticas (EPM) en las prácticas docentes en el proceso investigativo de su propia práctica. Las acciones investigativas y didácticas referidas anteriormente, han sido sedimentadas empírica y cognoscitivamente por el conjunto de evidencias, prácticas y saberes didácticos, meta-didácticos e investigativos, de la comunidad de la educación matemática del programa de formación de profesores de LEBEM, en los últimos veinte años (Lurduy, 2009).

\section{Motivaciones, justificaciones y problema de la investigación.}

De acuerdo con los más connotados investigadores y con las publicaciones nacionales e internacionales en educación matemática, estas sugieren la evidencia de diversidad, disparidad, multiplicidad de intereses y contenidos en las apuestas pedagógicas y didácticas de los programas de formación de profesores. Los autores consultados proponen investigar los temas asociados al desarrollo profesional del profesor, específicamente, la temática de la evaluación de las competencias desarrolladas en dichos programas (Lurduy, 2010).

Según los estudios reportados, el enfoque de las competencias está en el centro de las conceptualizaciones 
y decisiones de la política educativa nacional e internacional y constituyen una base fundamental para orientar el currículo, la docencia y la evaluación en todo el sistema educativo. De acuerdo con lo anterior, ellas son uno de los ejes en la acción formativa para los diversos niveles educativos, así como en los programas de formación de niños, jóvenes y profesores, en el imaginario de los planificadores y en el de muchos de los gestores de los programas de formación de profesores.

En lo referente a los antecedentes de investigación, según el estudio de Lurduy (2010), es evidente la poca atención a nivel nacional en esta temática de las competencias de los profesores, los formadores de profesores y aún más en la formación inicial de los profesores. Los documentos consultados aquí expresan el nivel de generalidad, diversidad, ambigüedad y poco desarrollo de las investigaciones, publicaciones y escritos sobre la temática de la formación de profesores de matemáticas, sobre los procesos de estudio didáctico y sobre las competencias desarrolladas en unos y otros.

\section{La situación problema de investigación}

En Colombia, el enfoque de competencias, a pesar de la exigencia institucional de su abordaje y de la desorientación de las comunidades educativas al respecto, tampoco ha sido suficientemente sustentado, investigado y estudiado, no existen evidencias que muestren la existencia de investigaciones sobre los programas de formación, tampoco sobre las competencias para el análisis y reflexión didáctica de los EPM desarrollados en esos programas. Igualmente, este enfoque, tampoco ha sido sustentado, estudiado, investigado, evaluado desde algunos de los modelos didácticos que son reconocidos por la comunidad de educadores matemáticos y que, en algunos casos, sustentan los mismos documentos que apoyan los enunciados de las políticas institucionales. Dado que el objeto de estudio de este trabajo de investigación son las competencias y su evaluación, nos preguntamos:

¿Qué conceptualización dela noción de competencia nos posibilita su evaluación en la formación de profesores?, ¿cuáles son las características de las competencias para el análisis, reflexión y semiosis didáctica de los profesores de matemáticas en formación?, ¿cómo podríamos evaluar esas competencias para el análisis, reflexión y semiosis didáctica de los estudiantes para serprofesoresde matemáticas, expresadas en los textos producidos por ellos mismos en estaformación? La investigación se propone la conceptualización y evaluación de las competencias para el análisis didáctico, la reflexión didáctica y para la construcción de significado sobre las acciones didácticas de los EPM, expresadas en los textos producidos por ellos como resultado de su proceso formativo como docentes de matemáticas para la educación básica.

\section{Los referentes teóricos de la investigación.}

El aporte y nuestra interpretación de EOS (Godino y Batanero, 1991 y 2011), al programa de investigación Rutas de Estudio y Aprendizaje en el aula de matemáticas (REA) (Lurduy, 2000-2005; Crisálida, 2002-2009) ha potenciado, actualizado los desarrollos teórico-metodológicos en y de esta investigación. En este trabajo adoptamos losmodelos:

$1^{\circ}$. Onto-epistemo de tipo triádico de la percepción, cognición y la significación en matemáticas y su didáctica - sobre bases semióticas de tipo pragmaticista-.

$2^{\circ}$. Epistemológico relacional, sobre las matemáticas y la reflexión didáctica - basado en presupuestos de complejidad antropológica y socio-constructivistas-.

$3^{\circ}$. Metodológico ético-pragmático, de la instrucción y los procesos de estudio matemático-didáctico en didáctica de la matemáticas, en la formación de profesores de matemáticas - sobre bases transmoderna y crítica del medio cultural iberoamericano-.

\section{Objetos, prácticas y problemas didácticos}

En la perspectiva semiótica adoptada, objeto semiótico es un elemento de la triada - objeto, representamen, interpretante-, así, su definición es sólo comprensible desde la coherencia con los principios epistemológicos peirceanos. En esta investigación, es esencial la diferenciación de las conceptualizaciones de los objetos didácticos y los meta-didácti$\cos$, pues tratamos con entidades, representaciones y 
significaciones diferentes, en el caso de un proceso de estudio didáctico, en donde tienen emergencia esos objetos didácticos.

Para nosotros una práctica didáctica se refiere a toda expresión, actuación y regulación que efectúa un profesor para resolver problemas didácticos - diseñar, gestionar y evaluar una secuencia de actividades sobre un tópico matemático escolar-. En la noción de práctica didáctica se articulan los aspectos actitudinales y axiológicos ligados a las situaciones-problema de tipo didáctico y por ello están ligados a la noción de competencia. El profesor (EPM) estudia y se compromete con aspectos didácticos que configuran otros objetos-procesos de segundo orden, que utiliza para construir conocimiento didáctico y pedagógico propio de su profesión y que evidencian su competencia profesional como docente.

El significado de un objeto didáctico - diseño, gestión y evaluación-lo caracterizamos como emergente del sistema de prácticas discursivas, operativas y normativas realizadas por una persona (EPM) al interior de una institución - la escuela o el aula-, en una práctica didáctica. Por lo tanto,estos significados se manifiestan en diferente grado con énfasis distintos, son complementarios entre sí y permiten la emergencia del significado de los objetos-procesos meta-didácticos de análisis, reflexión y semiosis didáctica, como solución a los problemas didácticos, propios de su profesión y hacen emerger y expresar su idoneidad profesional como profesor.

\section{Conceptualización de la competencia y la forma- ción de profesores.}

Nuestra conceptualización de la noción de competencia está acorde con nuestra interpretación de los principios de formación, los desarrollos de la propuesta formativa y los avances de investigación individual y colectiva en formación de profesores de matemáticas entre los años 2000 y 2012 en LEBEM. Igualmente, la enunciamos como relación de nuestra hipótesis abductiva, para configurar los elementos de significado de la competencia que serán abordados en este trabajo en el tema de la evaluación y que puede ser proyectada y utilizada para otras situaciones investigativas:

- Primeridad, lo óntico en la profesión ser profesor, $Q$, (querer-ser): Ser ontológico y analítico en el mundo de lo individual, colectivo y lo institucional, para la identidad, autoafirmación, auto-reconocimiento como profesores; consideración de la sensibilidad, afecto y motivación como potencia, motivación y dinámica de las formas de ser profesor de matemáticas, que hemos sintetizado como querer-ser (Q).

- Segundidad, lo epistémico en la profesión ser profesor, $P$, (querer/poder-ser/actuar): Poder-actuar epistemológico y reflexivo en la realidad didáctica-formativa emergente de la interacción entre el querer-ser/actuar y el poder-ser/actuar/saber en el mundo de lo individual, lo colectivo y lo institucional, que hemos sintetizado como poder-actuar $(P)$. Poder-ser/actuar/saber en la reflexión, configuración del saber, en la actualización desde el sentir, pensar y saber en la práctica didáctica y en la reflexión sobre las situaciones y problematizaciones en las relaciones profesor-saber-estudiante-entorno en las acciones didácticas.

- Terceridad, la metódica en la profesión ser profesor, C, (querer/poder/deber-ser/actuar/ saber): Deber/saber caracterizadopor las formas, hábitos, normas de ser/saber/saber en el mundo de lo individual, colectivo y lo institucional, que aquí hemos sintetizado como $s a$ ber-compromiso (C). Deber-ser/actuar/saber, como compromiso e implicación para la consideración del saber sobre las acciones didácticas y en la interacción formativa, así como para comprender el mundo de las interpretaciones, la intersubjetividad y la retroacción de las acciones y prácticas formativas, didácticas, y para las implicaciones individuales colectivas y sociales de su acción formativa como profesor de matemáticas. 


\section{Materiales y método para la evaluación de las competencias}

El proceso investigativo fue configurado, implementado y gestionado en el marco de las condiciones "naturales" de situaciones de tiempo y espacio de los desarrollos curriculares de la formación de profesores de matemáticas. Ello de acuerdo con las condiciones generales de determinación de los escenarios y actores (macro-caso), focalizadas de localización temática y de significado de las práctica didáctica textualizadas (meso-caso) y las situaciones específicas de evaluación de la información en las prácticas didácticas textualizadas como prácticas discursivas operativas y normativas (micro-caso).

\begin{tabular}{|c|c|c|c|c|c|c|}
\hline $\begin{array}{l}\text { Proceso } \\
\text { periodo }\end{array}$ & $\begin{array}{c}\text { Diseño, pilotaje, } \\
\text { validación } \mathrm{T}_{1}\end{array}$ & $\begin{array}{c}\text { Gestión } \\
\text { de la secuencia } \mathrm{T}_{1}\end{array}$ & $\begin{array}{l}\text { Evaluación de } \\
\text { la secuencia } T_{1} \\
\end{array}$ & $\begin{array}{l}\text { Implementación de } \\
\text { la investigación } \mathrm{T}_{2}\end{array}$ & $\begin{array}{c}\text { Desarrollo y } \\
\text { presentación de } \mathrm{T}_{3}\end{array}$ & $\begin{array}{c}\text { Base de datos } \\
\text { inicial }\left(\mathrm{B}_{0}\right)\end{array}$ \\
\hline |-2007 & $C_{1}, C_{2}$ & & & & & \\
\hline 2007-II & $C_{3^{\prime}} C_{4}$ & $C_{1^{\prime}} C_{2^{\prime}} C_{3^{\prime}} C_{4^{\prime}}$ & $C_{1}, C_{2}$ & $C_{1}, C_{2}$ & & \\
\hline | & $\mathrm{C}_{5^{\prime}} \mathrm{C}_{6^{\prime}} \mathrm{C}_{7^{\prime}} \mathrm{C}_{8^{\prime}} \mathrm{C}_{9^{\prime}}$ & $\mathrm{C}_{5^{\prime}} \mathrm{C}_{6^{\prime}} \mathrm{C}_{7^{\prime}} \mathrm{C}_{8^{\prime}} \mathrm{C}_{9}$ & $C_{3^{\prime}} C_{4^{\prime}} C_{5^{\prime}}$ & $\mathrm{C}_{3^{\prime}}, \mathrm{C}_{4}, \mathrm{C}_{5}$ & $C_{1}, C_{2}$ & \\
\hline \begin{tabular}{|l}
$2008-11$ \\
$2009-1$
\end{tabular} & $C_{10}$ & $C_{10}$ & $\mathrm{C}_{6^{\prime}} \mathrm{C}_{7^{\prime}} \mathrm{C}_{8^{\prime}} \mathrm{C}_{9}$ & $C_{6^{\prime}} C_{7^{\prime}} C_{8^{\prime}} C_{9^{\prime}}$ & $C_{3}, C_{4^{\prime}} C_{5^{\prime}}$ & $C_{1^{\prime}}, C_{2^{\prime}}, C_{3^{\prime}} C_{4^{\prime}}$ \\
\hline $\begin{array}{l}2009-I I \\
2010-I\end{array}$ & & & $C_{10}$ & $C_{10}$ & $C_{6^{\prime}} C_{7^{\prime}} C_{8^{\prime}} C_{9^{\prime}} C_{10}$ & $\begin{array}{l}C_{5^{\prime}} C_{6^{\prime}} C_{7^{\prime}} C_{8^{\prime}} \\
C_{9^{\prime}} C_{10}\end{array}$ \\
\hline
\end{tabular}

Tabla 1. Proceso de recolección de la información.

Fuente: elaboración propia

La tabla 1 resume el proceso llevado a cabo en la determinación de los casos, recopilación y delimitación de información y la configuración de la base inicial de datos conformada por los diez trabajos de grado de EPM de LEBEM, realizado entre los años 2007 y 2010.

\section{Materiales, casos y unidades de análisis.}

El total de EPM que participaron en esta investigación vivieron en su proceso de formación entre los años 2004 y 2010, y los casos específicos de EPM $\left(C_{i}\right)$ estudiados participaron entre los años 2004 y 2008 $\left(\mathrm{C}_{1}, \mathrm{C}_{2}, \mathrm{C}_{3}, \mathrm{C}_{4}\right) ; 2005$ y $2009\left(\mathrm{C}_{5}, \mathrm{C}_{6}\right)$; y 2006 y 2010 $\left(\mathrm{C}_{7}, \mathrm{C}_{8}, \mathrm{C}_{9}, \mathrm{C}_{10}\right)$, los textos $\mathrm{T}_{\mathrm{i}}$ corresponden: $\mathrm{T}_{1}$, a la unidad didáctica o informe escrito de la secuencia de actividades sobre el tópico matemático escolar en la práctica docente de los EPM; $\mathrm{T}_{2}$ corresponde a los videos y portafolios de los estudiantes de los respectivos cursos en donde los EPM realizan la investigación final; $\mathrm{y}_{3}$, el informe final de investigación de sus prácticas docentes, que es requisito para la obtención del título de licenciado en LEBEM y que en esta investigación fueron los textos analizados.
Una vez especificados los casos de los EPM e identificados los dieztextos $\left(T_{i 3}\right)$ producidos por ellos, la opción tomada, después de este proceso de inteligibilidad de situaciones y casosposibles, fue hacer una reducción a tres casos $\left(\mathrm{C}_{1}, \mathrm{C}_{5}, \mathrm{C}_{10}\right)$, que posibilitaron pilotar, validar y gestionar el proceso de recolección y análisis de la información en los textos y finalmente hacer un estudio específico detallado en el caso del texto $\mathrm{T}_{13}$ del caso $1\left(\mathrm{C}_{1}\right)$. Para nosotros es claro que lo fundamental y pertinente es la realización de "un análisis cualitativo del contenido expresado en los textos" desde una perspectiva ontosemiótica (Godino, 2002), complementada con la utilización de las semiótica peirceana como metodología para evaluar las competencias (Peirce, 1971).

\section{El método de la investigación.}

Inicialmente se determinaron algunos aspectos, principios y estrategias utilizadas y adaptadas por nosotros que permitían un adecuado manejo de la información dispuesta en los textos, se trata de algunos procedimientos y estrategias comunes y complementarias 
de: la Teoría fundamentada (TFD) (Glaser y Strauss, 1967; Strauss y Corbin, 1990, 2002); el Análisis cualitativo de contenido (ACC) (Bardin, 1986; Andreu, 2009); y el Análisis Semiótico de Textos (AST) (Abril, 1994; Fontanille 2004).

La figura 1 muestra los elementos que se consideraron pragmáticamente como comunes de las técnicas utilizadas para la recolección y tratamiento de la información- ACC-TFD-AST, - determinación de las unidades de análisis; elaboración de criterios para el tratamiento de la información, codificación, categorización, plantillas de registro de información-(Vasilachis, 2005). La figura 1 también representa y sintetiza el diseño, la gestión y la evaluación del proceso metodológico en la estrategia de movilidad, flexibilidad y articulación metodológica para tratar los casos de los EPM $\left(\mathrm{C}_{\mathrm{i}}\right)$ y los textos estudiados $\left(\mathrm{T}_{\mathrm{ij}}\right)$, donde con los subíndices indicamos que i representa el caso del estudiante y $\mathrm{j}$ el tipo de texto abordado, en resumen $\left(C_{i}-T_{i j}\right)$.

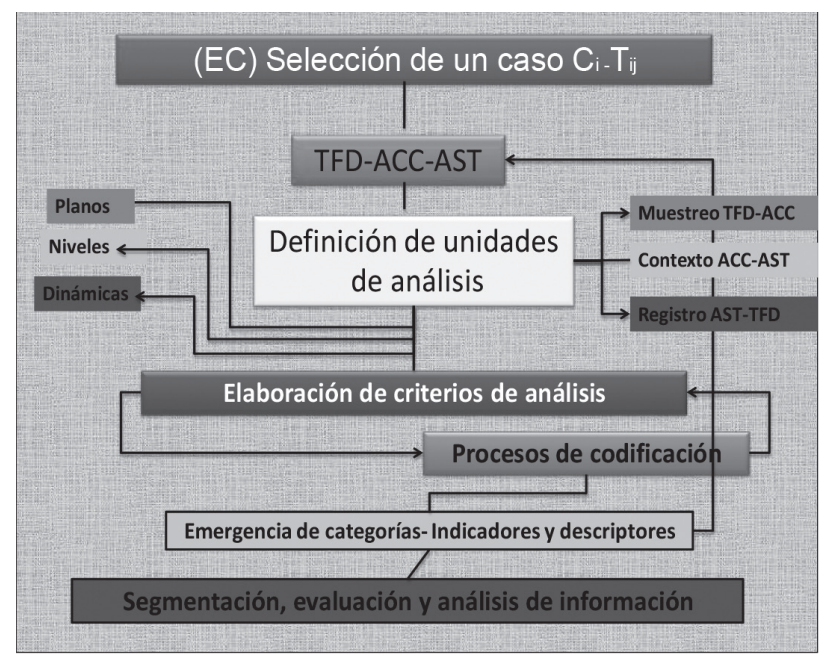

Figura 1. Determinación del proceso metodológico.

Fuente: elaboración propia

Para una primera recolección, delimitación y reducción de los datos, combinamos y adaptamos algunas de las estrategias metodológicas de TFD con algunos de los aspectos pertinentes de ACC, que posibilitaron homogeneizar y tratar la gran cantidad de información; para el tratamiento, delimitación y focalización de la información, utilizamos la combinación de algunos de los procedimientos del ACC con los aspectos de AST que le eran complementarios, de manera que se pudieran generar las condiciones metodológicas y conceptualmente adecuadas para el tratamiento de los textos; para el proceso de análisis específico de la información en los textos, que permitiera la evaluación de las competencias expresadas en los textos, combinamos convenientemente aspectos del análisis semiótico de los textos con aspectos técnicos de TFD con los que pudimos interpretar, inferir y abducir los resultados del proceso de evaluación de las competencias expresadas en los textos de los EPM.

\section{Metodología y condiciones para la evaluación de las competencias.}

Primero, se determinaron los casos de los EPM estudiados y los textos producidos por ellos, para delimitar la información se redujo a un subconjunto de textos, se configuraron y determinaron las organizaciones y contenidos textuales más representativos de LEBEM. Al estudiar la organización, registro e interpretación de los textos, apostamos por establecer diferencias en las descripciones y narraciones que garanticen la pertinencia, validez y fiabilidad de la información - planos de configuración textual, niveles de expresión de las relaciones didácticas y dinámicas de descripción de las interacciones didácticas en cada clase-, por lo tanto, los análisis y las informaciones resultantes en esta parte de la investigación son generales, se refieren al contenido organizativa de los textos, a las caracterizaciones básicas y determinantes de unas condiciones mínimas pero suficientes para que cada texto sea tenido en cuenta como fuente final de información de esta investigación.

- Procedimiento de primera reducción de la información:Usamos el proceso que denominamos codificación abierta y registro en cuadernos y memorandos de anotaciones en los mismos textos, registramos la recolección de la información en los textos. De manera que, segmentando y codificando la información textualizada, se pudo reducir, analizar, delimitar y diferenciar la información contenida en los textos. Este procedimiento de evaluación de la información general en los textos 
lo implementamos en los diez casos "seleccionados" de LEBEM, para posteriormente reducirlos a tres casos icónicos $\left(\mathrm{C}_{1}, \mathrm{C}_{5}, \mathrm{C}_{10}\right)$, tomando como criterio la cantidad, profundidad y calidad de la información en relación con los intereses de esta investigación.

La tabla 2 es un resumen del proceso de codificación de los textos y de la manera como en ellos se expresan los planos, niveles y dinámicas, como ya se dijo, estos son una expresión de la configuración triádica de la información y de su diferenciación que, en términos de la semiótica peirceana, relacionamos con la primeridad, segundidad y terceridad; se resaltan los casos que emergen como representativos de cada una de las categorizaciones los indicadores y descriptores propuestos para analizar el contenido de los textos.

\begin{tabular}{|c|c|c|c|}
\hline & PRIMERIDAD & SEGUNDIDAD & TERCERIDAD \\
\hline $\begin{array}{l}\text { PLANOS } P_{i} \\
\text { Configuración } \\
\text { textual }\end{array}$ & $\begin{array}{l}\text { Descriptivo-gramatical }\left(P_{1}\right) \\
{\left[C_{1^{\prime}}, C_{2^{\prime}} C_{3^{\prime}} C_{4^{\prime}} C_{5^{\prime}} C_{6^{\prime}} C_{7^{\prime}} C_{8^{\prime}} C_{9^{\prime}}, C_{10^{\prime}}\right]}\end{array}$ & $\begin{array}{l}\text { Semántico-situacional }\left(\mathrm{P}_{2}\right) \\
{\left[\mathrm{C}_{1^{\prime}}, \mathrm{C}_{3^{\prime}} \mathrm{C}_{5^{\prime}} \mathrm{C}_{6^{\prime}} \mathrm{C}_{10^{\prime}}\right]}\end{array}$ & $\begin{array}{l}\text { Pragmático-significativo }\left(\mathrm{P}_{3}\right) \\
{\left[\mathrm{C}_{1}, \mathrm{C}_{2^{\prime}} \mathrm{C}_{3^{\prime}} \mathrm{C}_{4^{\prime}} \mathrm{C}_{5^{\prime}} \mathrm{C}_{7^{\prime}} \mathrm{C}_{10}\right]}\end{array}$ \\
\hline $\begin{array}{l}\text { NIVELES } N_{i} \text { Relación } \\
\text { didáctica }\end{array}$ & $\begin{array}{l}\text { Profesor-saber }\left(\mathrm{N}_{1}\right) \\
{\left[\mathrm{C}_{1^{\prime}} \mathrm{C}_{2^{\prime}} \mathrm{C}_{3^{\prime}} \mathrm{C}_{4^{\prime}} \mathrm{C}_{5^{\prime}} \mathrm{C}_{6^{\prime}} \mathrm{C}_{7^{\prime}} \mathrm{C}_{8^{\prime}} \mathrm{C}_{9^{\prime}} \mathrm{C}_{10^{\prime}}\right]}\end{array}$ & $\begin{array}{l}\text { Profesor-entorno }\left(\mathrm{N}_{2}\right) \\
{\left[\mathrm{C}_{3^{\prime}} C_{4^{\prime}} C_{5^{\prime}} C_{6^{\prime}} C_{10^{\prime}}\right]}\end{array}$ & $\begin{array}{l}\text { Profesor-estudiante }\left(\mathrm{N}_{3}\right) \\
{\left[\mathrm{C}_{1}, \mathrm{C}_{2^{\prime}}, \mathrm{C}_{5^{\prime}} \mathrm{C}_{6^{\prime}} \mathrm{C}_{7^{\prime}}, \mathrm{C}_{8^{\prime}} \mathrm{C}_{9^{\prime}} \mathrm{C}_{10}\right]}\end{array}$ \\
\hline $\begin{array}{l}\text { DINÁMICAS } \\
\text { Dilnteracción } \\
\text { didáctica }\end{array}$ & $\begin{array}{l}\text { Validación-Acción }\left(D_{1}\right) \\
{\left[C_{1^{\prime}}, C_{2^{\prime}} C_{3^{\prime}} C_{4^{\prime}} C_{5^{\prime}} C_{6^{\prime}} C_{7^{\prime}} C_{8^{\prime}} C_{9}\right]}\end{array}$ & $\begin{array}{l}\text { Validación-Formulación }\left(D_{2}\right) \\
{\left[C_{1}, C_{3,} C_{4^{\prime}} C_{5^{\prime}} C_{6}\right]}\end{array}$ & $\begin{array}{l}\text { Validación-institucionalización }\left(D_{3}\right) \\
{\left[C_{1}, C_{5}, C_{6^{\prime}} C_{7^{\prime}}, C_{8^{\prime}}, C_{9^{\prime}}\right]}\end{array}$ \\
\hline
\end{tabular}

Tabla 2. Reducción y organización de la información en $\left(T_{i 3}\right)$.

Fuente: elaboración propia

Finalmente, de lo que se trataba esta fase del proceso metodológico era del reconocimiento de criterios de diferenciación-principio de evaluación orientativa-del contenido de los diferentes textos, así como de generar e inferir en esos textos un espacio de posible convencionalidad, cierto grado de homogeneidad y representatividad que nos posibilite efectivamente la interpretación de la información a partir del análisis de lo enunciado en los textos.
Todo lo narrado anteriormente y configurado en la tabla 3, evidencia y soporta la decisión de realizar todo el proceso analítico específico para que se pilote en $\mathrm{C}_{10}$, se valide en $\mathrm{C}_{5}$ y se aplique el análisis focal de la información para el caso de $\mathrm{C}_{1}$, en el texto $\mathrm{T}_{13}$, pues éste evidencia ser el caso más representativo y simbólico de la producción de los textos de los EPM en LEBEM. Que denominamos focalización de la información.

\begin{tabular}{|c|c|c|c|}
\hline (TFD-ACC-AST) & $\left(T_{i 3}\right)$ & $\left(T_{i 3}\right)$ & $\left(T_{i 3}\right)$ \\
\hline $\begin{array}{l}\text { PLANOS de configuración } \\
\text { textual (querer-ser) }\end{array}$ & $\begin{array}{l}\text { Descriptivo-gramatical } \\
\left(\mathrm{P}_{1}\right) \\
{\left[\mathrm{C}_{1}, \mathrm{C}_{5}, \mathrm{C}_{10^{\prime}}\right]}\end{array}$ & $\begin{array}{l}\text { Semántico-situacional } \\
\left(\mathrm{P}_{2}\right) \\
{\left[\mathrm{C}_{1^{\prime}}, \mathrm{C}_{5^{\prime}}, \mathrm{C}_{10^{\prime}}\right]}\end{array}$ & $\begin{array}{l}\text { Pragmático-significativo } \\
\left(\mathrm{P}_{3}\right) \\
{\left[\mathrm{C}_{1^{\prime}}, \mathrm{C}_{5^{\prime}} \mathrm{C}_{10^{\prime}}\right]}\end{array}$ \\
\hline $\begin{array}{l}\text { NIVELES de relación didáctica } \\
\text { (poder-ser-hacer) }\end{array}$ & $\begin{array}{l}\text { Profesor-saber } \\
\left(\mathrm{N}_{1}\right) \\
{\left[\mathrm{C}_{1}, \mathrm{C}_{5}, \mathrm{C}_{10}\right]} \\
\end{array}$ & $\begin{array}{l}\text { Profesor-entorno } \\
\left(\mathrm{N}_{2}\right) \\
{\left[\mathrm{C}_{1}, \mathrm{C}_{5}\right]} \\
\end{array}$ & $\begin{array}{l}\text { Profesor-estudiante } \\
\left(\mathrm{N}_{3}\right) \\
{\left[\mathrm{C}_{1}, \mathrm{C}_{5^{\prime}}\right]} \\
\end{array}$ \\
\hline $\begin{array}{l}\text { DINÁMICAS de la interacción } \\
\text { didáctica (deber-ser-hacer) }\end{array}$ & $\begin{array}{l}\text { Validación-Acción } \\
\left(D_{1}\right) \\
{\left[C_{5}\right]} \\
\end{array}$ & $\begin{array}{l}\text { Validación-Formulación } \\
\left(D_{2}\right) \\
{\left[C_{1^{\prime}}, C_{5^{\prime}}\right]} \\
\end{array}$ & $\begin{array}{l}\text { Validación-institucionalización } \\
\left(\mathrm{D}_{3}\right) \\
{\left[\mathrm{C}_{5^{\prime}} \mathrm{C}_{10^{\prime}}\right]} \\
\end{array}$ \\
\hline
\end{tabular}

Tabla 3. Primera reducción, organización de la información en $\left(\mathrm{T}_{\mathrm{i} 3}\right)$.

Fuente: elaboración propia

La tabla 3 se propone un resumen de la reorganización y sistematización de los análisis e inferencias realizadas en este ámbito de la investigación para un análisis generalizado de la organización textual de información, es conducente a las decisiones de desarrollo y gestión de la investigación. 
- Procedimiento de delimitación y focalización de la información: Esta segunda fase de aplicación metodológica consistió en que, por medio de la lectura intensiva de tipo interpretativo, identificamos y codificamos los diferentes planos de configuración semiótica, según se refieran a las temáticas del diseño, gestión y evaluación $\left(\mathrm{P}_{\mathrm{i}}\right)$ de la acción didáctica. Posteriormente, reiteramos el proceso para los diferentes niveles de configuración del eje de significado didáctico, niveles de análisis, reflexión y semiosis didáctica $\left(\mathrm{N}_{\mathrm{i}}\right)$ identificados en los textos. Finalmente, determinar los sistemas de prácticas en la situación y contexto de realización de las acciones didácticas — significado didáctico-enunciadas en los textos identificándolas en los textos y codificándolas como prácticas discursivas, operativas y normativas $\left(\mathrm{D}_{\mathrm{i}}\right)$.

En este tipo de procedimiento se utiliza la codificación axial,que se hizo, primero, en cada una de las unidades de contexto analizadas, identificando los planos, los niveles y las dinámicas - lectura extensiva, en búsqueda de los atractores de significado-. Posteriormente, de forma escalonada - relectura intensiva-se hace una especie de jerarquización significativa de la información desde las condiciones iniciales-limitantes (planos), después por los niveles focales (niveles), después con el micro-nivel de determinación específica (dinámicas).

\section{- Identificación focal de la información en los} textos: Consideramos al conjunto de textos como textos local y focalmente homogéneos y, en este sentido, el texto seleccionado es icónico de la producción de los informe finales de investigación e indicativo del contenido de la información necesaria y suficiente para poder implementar el proceso metodológico de evaluación de las competencias. El texto estudiado, es "individual, particular y focal", es decir, escrito por un sujeto, en situaciones diferentes a los otros textos, pero con características contextuales comunes a los individuos "productores" de los textos.

Consistentes con nuestra apuesta de la semiótica como una metodología para el análisis de textos, nuestra hipótesis abductiva, es, por un lado, este ámbito de la investigación nos permite identificar y describir focalmente los elementos primarios de significado del análisis, reflexión y semiosis didáctica. Por otro lado, estos elementos de significado, funcionarían como filtros óntico-epistémicos de tipo regulativo y operativo para evaluar las competencias de los EPM evidenciadas en los tipos y sistemas de prácticas discursivas, operativas y normativas, que fueron textualizadas.

Según nuestra interpretación de este ámbito de la investigación después de la primera lectura extensiva, las unidades de análisis correspondieron a las que evidenciaron contextualización de las temáticas y de esa manera se segmentaron y clasificaron los textos correspondientes al contenido de dicha información:

-Objetos didácticos, $\mathrm{P}_{\mathrm{i}}$ (diseño, gestión evaluación).

-Objetos-procesos didácticos de investigación, $\mathrm{N}_{\mathrm{i}}$ (análisis, reflexión, semiosis didácticas).

-Tipos de prácticas sobre objetos didácticos de investigación, $\mathrm{D}_{\mathrm{i}}$ (prácticas discursivas operativas y normativas).

En nuestro caso, identificar, interpretar y recuperar los elementos de significado de los atractores semióticos, los criterios de evaluación de ellos mismos, como indicadores de la presencia de las competencias de un EPM, cuando ellos abordan una práctica didáctica.

- Inferencias del análisis focalizado de la información: En un apartado anterior, identificamos, caracterizamos y describimos los atractores de significado, a partir de las conceptualizaciones iniciales sobre los objetos didácticos ylos meta-didácticos. Así mismo, se evidenció la actualización de las competencias en EPM, expresadas en los textos analizados. También certificamos la identificación, caracterización y descripción del sistema de prácticas discursivas, operativas y normativas. Por lo tanto inferimos que podemos describir y caracterizar - para poder evaluar-, por un lado, los elementos de significado, y por otro, la emergencia del significado de sentido que construyen los EPM. 
Consistentes con nuestra apuesta metodológica, la textualización de las competencias aún no es certificativa de los elementos de significado de las competencias, de sus niveles y tipos de significado de expresión semiótica en los textos, así como de sus descriptores cualitativos, para lo que tendremos que apostar a evaluarlas en el siguiente ámbito de investigación. Por un lado, al certificar la textualización y expresión de las competencias para el análisis, reflexión y semiosis didácticas de los EPM y, en este sentido, describirlas focalmente, estas han sido evidenciadas en los tipos y sistemas de prácticas discursivas, operativas y normativas.

Por otra parte, las unidades de análisis, que denominamos unidades de contexto, son certificativas de la existencia física de la condiciones requeridas para evaluar las competencias, pues estas unidades de análisis correspondieron a las que evidenciaron contextualización de las temáticas -objetos didácticos- $\mathrm{P}_{\mathrm{i}}$ (diseño, gestión evaluación); objetos-procesos didácticos de investigación $\mathrm{N}_{\mathrm{i}}$ (análisis, reflexión, semiosis didácticas); situación-tipos de prácticas sobre objetos didácticos de investigación $\mathrm{D}_{\mathrm{i}}$ (prácticas discursivas operativas y normativas).

Como lo muestran los diferentes apartados en $\mathrm{T}_{13}$, el análisis didáctico, la reflexión didáctica y la semiosis didáctica emergenteen una interacción didáctica son icónicas, indiciales y simbólicas correspondientemente de la relación triádica entre las caracterizaciones fenoménicas de las competencias: (querer-ser/ actuar/saber),(querer-poder-ser/actuar/saber), (querer-poder-deber-ser/actuar/saber). Estas configuraciones triádicas utilizadas en los niveles y tipos de signos de expresión semiótica son una herramienta metodológica para la evaluación de las competencias de los EPM.

\section{Resultados del proceso específico de evaluación de las competencias.}

El proceso específico de análisis de la información lo hacemos en el texto $\left(T_{13}\right)$, en él se le da continuidad al proceso de flexibilidad, combinación y articulación de los procedimientos para analizar y evaluar textos escritos. Se trata de la aplicación de nuestra interpretación de algunas nociones y herramientas del análisis ontosemiótico, complementada con la adaptación hecha por nosotros de la perspectiva semiótica peirceana. El procedimiento de segmentación, organización y sistematización de la información en los textos, lo hacemos con nuestra interpretación, adaptación y aplicación de las técnicas propuestas por Abril (1994). Se trata de la utilización de los procesos, codificación selectiva de la información, registros de comentarios en los textos mismos, y la posterior dimensionalización cualitativa de la información en rangos y rasgos de esa información.

\section{Proceso de reducción de la información.}

Como ya se enunció en las unidades de análisis $\left(\mathrm{T}_{13}\right)$, analizamos y evaluamos en las unidades de generales de registro $\left(\tau_{13}\right)$ el sentido de las acciones didácticas como el objeto emergente del subsistema de prácticas textualizadas, discursivas, operativas y normativas, identificación de planos de expresión ontosemiótica, - escalonamiento de sentido en tipos de prácticas-. Posteriormente, en las unidades focales de registro $\left[\left(\mathbf{U}_{13}\right)^{\mathrm{D}} ;\left(\mathbf{U}_{13}\right)^{\mathrm{O}} ;\left(\mathbf{U}_{13}\right)^{\mathrm{N}}\right]$, caracterizamos indicadores de expresión semiótica de las competencias, como niveles y tipos de signos que evidencian la presencia de los elementos de significado de las competencias, caracterización de niveles de expresión semiótica, —ordenamiento de la expresión semiótica en niveles y tipos de signos-. Se determina y específica, primero, el sentido de las acciones didácticas, después, se caracteriza la expresión semiótica de las competencias mediante la identificación, caracterización de niveles de expresión semiótica; finalmente, se realiza la identificación, conceptualización y caracterización de los descriptores semióticos de los tipos de signos (Niño, 2007, b).

Por lo anterior,se produce una secuencial recolección, reducción y organización selectiva $y$ significativa de los textos. ${ }^{22}$ Finalmente, en las unidades de específicas de registro $\left(\boldsymbol{u}_{13}\right)$, procedimos a la

3 Tal como se entiende en la semiótica, según Fontanille,(2006), la lectura (metodología) semiótica es una práctica especializada e interpretativa de la objetividad y procesualidad de un fenómeno, en el sentido del estudio de la producción e interpretación del sentido en contextos específicos y determinados, en este caso, los textos que resultan de una acción didáctica. 
determinación de las dinámicas de expresión de las competencias y en ellas, se evalúan los descriptores cualitativos de los tipos de signos en términos de los elementos de significado de los objetos didácticos y meta-didácticos - grados de expresión semiótica de la competencia en descriptores cualitativos-.

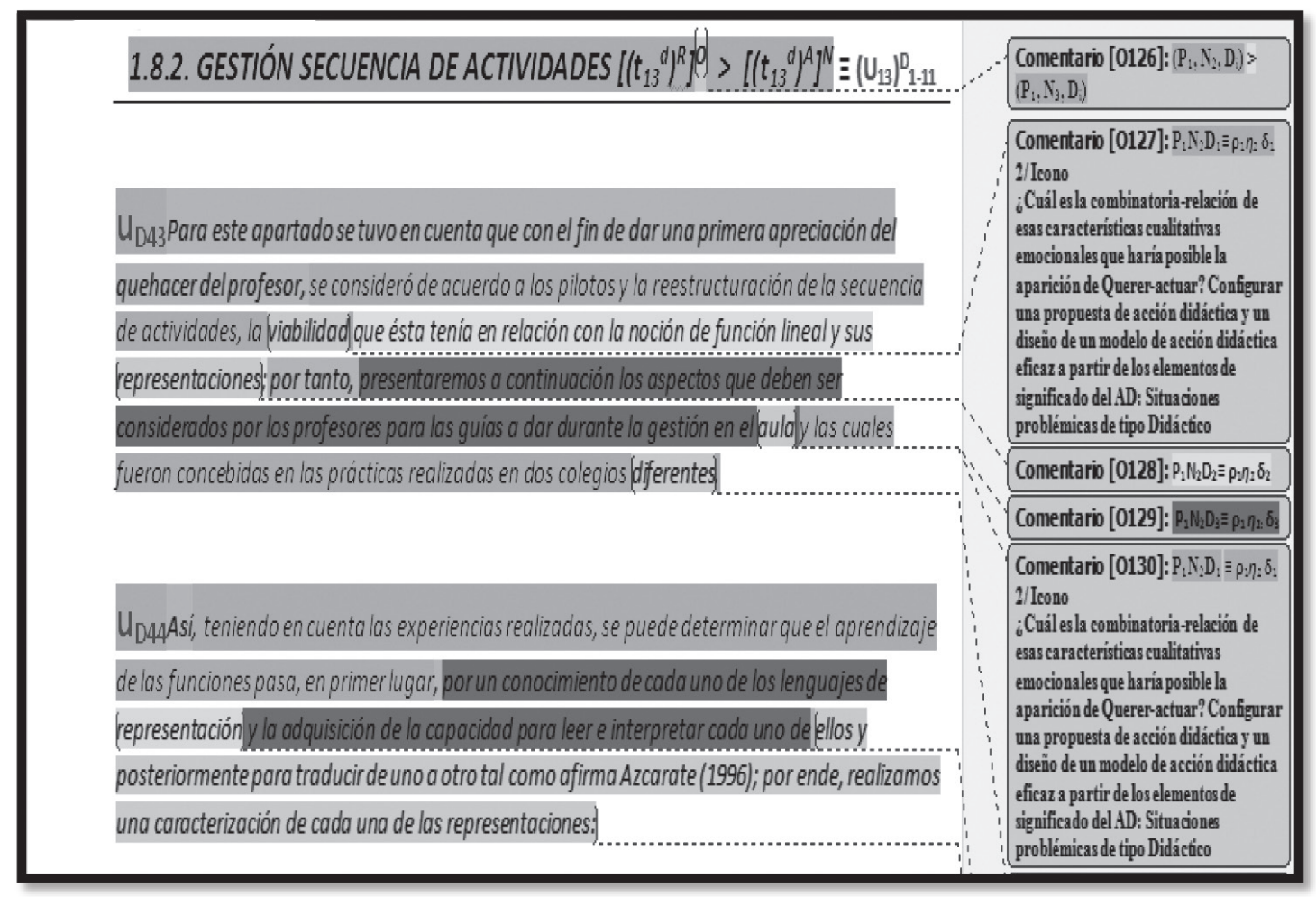

Figura 2. Unidades específicas de registro y análisis de la información.

Fuente: elaboración propia

La figura 2 muestra un ejemplo del resultado de determinación de las unidades específicas de registro $\left[\left(\mathbf{u}_{43}\right)\right.$; $\left(\mathbf{u}_{44}\right)$ ]. Igualmente, muestra ejemplos del proceso implementado en los textos mismos para registrar códigos y, finalmente, muestra un ejemplo de la configuración de los instrumentos para análisis e interpretación de la información-memorandos de comentarios de análisis de la información-. Así mismo, se muestra el registro de los comentarios con respecto al tipo de descriptor de la competencia y, en correspondencia, los comentarios de análisis, interpretación e inferencias del respectivo descriptor del objeto meta-didáctico.
El procedimiento evaluativo de las competencias, en términos de las dinámicas de sentido expresadas en los textos, consistió en separar las unidades específicas de análisis, interpretar las frases, proposiciones y términos en dichas unidades, identificando el nivel semiótico y el tipo de signo. Posteriormente, caracterizamos el tipo de expresión relacionándola con los elementos de significado de los objetos-procesos meta-didácticos, para, finalmente, asignarle un descriptor cualitativo que sería representativo, certificativo y nominativo del elemento de significado de la competencia. 


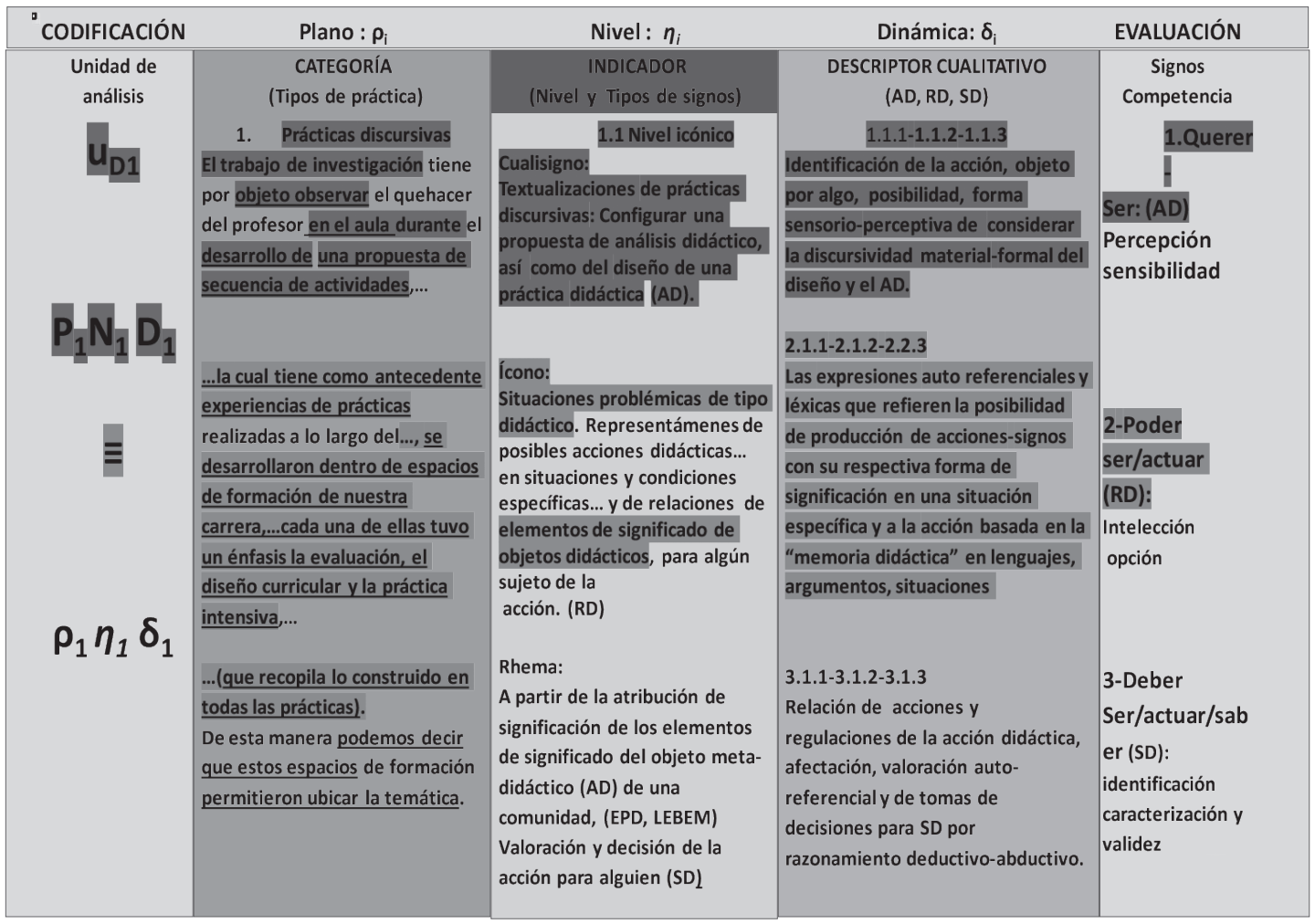

Figura 3. Ejemplo de unidades específicas de análisis.

Fuente: elaboración propia

En la figura 3 se muestra el análisis realizado para cada unidad específica de análisis, en este caso, para las prácticas discursivas: identificación de la unidad de análisis, segmentación e identificación de frases, términos y enunciados del respectivo lexema, (subrayado y colores). Identificación del nivel y tipo de signo de expresión de la competencia; identificación y descriptor cualitativo del correspondiente elemento de significado del objeto meta-didáctico correspondiente - en esta unidad específica, análisis didáctico-y evaluación del elemento de significado de la competencia y del descriptor cualitativo emergente.

\section{Análisis específico de la información.}

La interpretación, pragmática, verificativa ontosemiótica en este ámbito de la investigación tuvo como referencia las interpretaciones-análisis de las "superficies textuales" y de presencia de significado de los objetos didácticos en los textos, estudiadas en el primer ámbito de aplicación metodológica. También tiene como base de regulación-interpretación las reflexiones-abducciones del estudio inferencial de los "contenidos textuales" de tipo semántico-situacional y los ejes de significación de los objetos meta-didácticos, estudiadas en el segundo ámbito de aplicación métodológica en este trabajo. Las principales caracterizaciones e inferencias resultado del análisis específico de la información las referenciamos de la siguiente manera:

- Atractores semióticos: De manera general proponemos que, en un sistema semiótico de tipo didáctico, denominamos "atractor semiótico" al conjunto de signos que, con cierta constancia, relativa reiteración y operación voluntaria, ocasionan permanencia en la memoria de otros signos (interpretantes dinámicos). Dichos atractores tienen una función semiótica en la adopción de una acción, una 
rutina de acción o un rol de interacción didáctica y se encuentran disponibles para compararse y contrastarse con unos determinados conjuntos de signos percibidos - en este caso los elementos de significado de las competencias: querer/poder/deber y ser/actuar/saber, propuestos, actualizados yregulados para otras interacciones didácticas-.

- Contenido de los textos: La intencionalidad pragmática de verificación y evaluación semiótica del "fondo de los textos", hace de esta evaluación complementaria, dialógica, pero radicalmente distinta de las realizadas anteriormente en los otros ámbitos de aplicación metodológica de este trabajo. Por lo tanto, es importante insistir aquí sobre la necesidad de descripción densa de la información de tipo semiótico, para la estructuración de significado, en la emergencia de las "caracterizaciones teóricas" en los datos, en la estructuración del sentido en el contenido de los datos y sobre la dimensionalización ontosemiótica de los contenidos significativos con sentido en los textos y subtextos.

- La semiótica como metodología: La onto-epistemo-semiótica referida y utilizada como "metodología semiótica",nos habla del contexto-situación-problema del texto y de las relaciones complejas de tipo contextual. Se refiere a la relación triádica objeto-sujeto-método. La semiótica como práctica metodológica se trata aquí de una reconstrucción narrativa interpretativa, argumentativa e inferencial de los textos, como una praxis especializada de lectura - extensiva, intensiva, densa-de esos textos específicos, para evaluar las competencias de análisis, reflexión y semiosis didáctica en expresadas en los textos de los EPM.

- La competencia: Los textos permitieron, recursiva, dialogal y complementariamente, identificar niveles de expresión semiótica en tipos de signos y caracterizar los descriptores de expresión de la competencia de forma modal (querer/poder/saber-ser/actuar/deber) y sistémica (estructurada/triádica/compleja). El término modal y sistemático se refiere a la adhesión continua y rigurosa que expresamos respecto a un enunciado a lo largo del discurso en el texto y por tanto los conceptos/ estrategias frente al texto de distancia, transparencia y tensión son característicos de esa modalización sistemática.

La vía modal y sistemática de la identificación de dichos atractores nos permitió la evaluación del contenido del "fondo de los textos", con una intencionalidad evaluativa de tipo validativa, certificativa y normativa, argumentada y sustentada en los procedimientos para analizar textos mediante un abordaje metodológico de tipo semiótico.

- La evaluación de la información en las unidades específicas de análisis: En este micro-nivel de análisis de los textos de los EPM, dichos atractores semióticos configuran el espacio de configuración y dimensionalización significativa de la información -significado y sentido en términos de tipos de prácticas-, para las condiciones de determinación específicas de producción de los textos, en los casos específicos de los EPM.

\begin{tabular}{|c|c|c|c|c|c|c|}
\hline \multicolumn{7}{|c|}{ EVALUACIÓN DE LA INFORMACIÓN } \\
\hline Categoría & \multicolumn{3}{|c|}{ Indicador } & \multicolumn{3}{|c|}{ Descriptor } \\
\hline $\begin{array}{l}\text { Sentido } \\
\text { PLANO } \rho_{\mathrm{i}}\end{array}$ & \multicolumn{3}{|c|}{$\begin{array}{l}\text { Niveles de expresión semiótica } \\
\text { NIVEL } \eta_{i}\end{array}$} & \multicolumn{3}{|c|}{$\begin{array}{l}\text { Descriptores de signos de } \\
\text { DINÁMICA } \delta_{i}\end{array}$} \\
\hline $\begin{array}{c}\text { subtipos de } \\
\text { prácticas }\end{array}$ & \multicolumn{3}{|c|}{ Tipos de signos } & $A D$ & $\mathrm{RD}$ & SD \\
\hline \multirow{3}{*}{$\begin{array}{l}\text { 1.Prácticas } \\
\text { discursiva } \\
\text { (querer-ser) }\end{array}$} & \multirow{3}{*}{$\begin{array}{c}\underline{1 .} \\
\text { Cualisigno }\end{array}$} & \multirow{3}{*}{$\begin{array}{c}\underline{2} . \\
\text { Icono }\end{array}$} & \multirow{3}{*}{$\begin{array}{c}\underline{3 .} \\
\text { Rhema }\end{array}$} & 1.1.1.1 & 2.1.1. & 3.1.1. \\
\hline & & & & 1.1.2. & 2.1.2. & 3.1.2 \\
\hline & & & & 1.1.1. & 2.1. $\underline{3}$ & 3.1 .3 \\
\hline \multirow{3}{*}{$\begin{array}{c}\text { 2.Prácticas } \\
\text { operativas } \\
\text { (poder-actuar) }\end{array}$} & \multirow{3}{*}{$\begin{array}{c}4 . \\
\text { Sinsigno }\end{array}$} & \multirow{3}{*}{$\begin{array}{c}\underline{5 .} \\
\text { Índice }\end{array}$} & \multirow{3}{*}{$\begin{array}{c}\underline{6} . \\
\text { Decisigno }\end{array}$} & 1.2.4 & 2.2.4 & 3.2. \\
\hline & & & & 1.2.5 & 2.2.5 & 3.2.5 \\
\hline & & & & 1.2.6 & 2.2.6 & 3.2.6 \\
\hline \multirow{3}{*}{$\begin{array}{c}\text { 3.Prácticas } \\
\text { normativas } \\
\text { (Deber-saber) }\end{array}$} & \multirow{3}{*}{$\begin{array}{c}\underline{7 .} \\
\text { Legisigno }\end{array}$} & \multirow{3}{*}{$\begin{array}{c}\stackrel{8 .}{\text { Símbolo }} \\
\end{array}$} & \multirow{3}{*}{$\begin{array}{c}9 . \\
\text { Argumento }\end{array}$} & 1.3.7 & 2.3.7 & 3.3.7 \\
\hline & & & & 1.3.8 & 2.3.8 & 3.3 .8 \\
\hline & & & & 1.3.9 & 2.3.9 & 3.3 .2 \\
\hline
\end{tabular}

Figura 4. Evaluación de la información.

Fuente: elaboración propia

La figura 4 resume y representa la aplicación y utilización de los elementos de significado de los objetos meta-didácticos a las unidades específicas de análisis, relacionándolos con los elementos de significado de la competencia; los niveles y tipos de expresión semiótica en los textos, de los que se infieren, los descriptores cualitativos de expresión de las competencias. 
Con la sistematización anterior se puede identificar, describir ydenominar los tipos de elementos primarios de la competencia, expresados en los tipos de prácticas textualizadas y relacionadas con los indicadores de los respectivos signos. También se corresponde con los descriptores cualitativos de la expresión de la competencia y permiten inferir que los textos estudiados representan una síntesis simbólica de la expresión de $€ \Xi$ [querer/poder/deber-ser/ actuar/saber] de los EPM. Esta evaluación, a su vez, fue actualizada y realizada con la información.

Instrumentos de sistematización y análisis de la información: Finalmente, emergen en la configuración de análisis específico de la información; las figuras $4,5,6,7$, y 8 resumen el conjunto de categorías, indicadores y descriptores utilizados para el análisis interpretación de la información, en la perspectiva de evaluar la competencia, en este caso, los elementos de significado de la competencia para el AD, RD y SD.

\begin{tabular}{|c|c|c|c|}
\hline$(\mathrm{Q}, \mathrm{P}, \mathrm{C})$ & $\begin{array}{l}\text { Prácticas discursivas de } \\
\text { AD: posibilidad } \\
\text { Forma/cualidad/diseño }\end{array}$ & $\begin{array}{l}\text { Prácticas operativas de RD: } \\
\text { existencia } \\
\text { Acción/comportamiento/gestión }\end{array}$ & $\begin{array}{l}\text { Prácticas normativas de SD: necesidad } \\
\text { Interpretación/evaluación/semiosis }\end{array}$ \\
\hline $\begin{array}{l}\text { Nivel icónico: } \\
11 \equiv \eta 1\end{array}$ & $\begin{array}{l}\text { Querer -ser } \\
\text { Cualisigno: forma de la } \\
\text { forma } \\
\text { Intelección /disposición } \\
\text { de posibles componentes } \\
\text { cualitativos para } \\
\text { propuesta de práctica } \\
\text { didáctica (AD). } \\
\text { 1.1.1, 1.1.2, 1.1.3 }\end{array}$ & $\begin{array}{l}\text { Querer-Actuar/hacer } \\
\text { Icono: existencia de la forma } \\
\text { Configurar una ícono de una } \\
\text { acción y/o comportamiento en la } \\
\text { gestión de una práctica didáctica } \\
\text { (RD). } \\
2.1 .1,2.1 .2,2.1 .3\end{array}$ & $\begin{array}{l}\text { Querer-compromiso } \\
\text { Rhema: valor de la forma } \\
\text { Norma, orden, advertencia constitutivas } \\
\text { de componentes cualitativos de una } \\
\text { práctica didáctica (SD). } \\
3.1 .1,3.1 .2,3.1 .3\end{array}$ \\
\hline $\begin{array}{l}\text { Nivel indicial: } \\
12 \equiv \eta 2\end{array}$ & $\begin{array}{l}\text { Poder-ser } \\
\text { Sinsigno: forma de la } \\
\text { existencia. Identidad } \\
\text { existencial de } \\
\text { componentes cualitativos } \\
\text { para la acción didáctica } \\
\text { en la posible utilización } \\
\text { del AD } \\
\text { 1.2.4, 1.2.5, 1.2.6 }\end{array}$ & $\begin{array}{l}\text { Poder-actuar/hacer } \\
\text { Índice:, relacionar un fenómeno } \\
\text { didáctico determinado mediante } \\
\text { un índice de una RD y/o } \\
\text { comportamiento en la práctica } \\
\text { didáctica } \\
2.2 .4,2.2 .5,2.2 .6\end{array}$ & $\begin{array}{l}\text { Poder-saber/compromiso } \\
\text { Dicisigno: existencia del valor o } \\
\text { representar convencionalmente un } \\
\text { fenómeno mediante un símbolo de una } \\
\text { SD en la gestión de una práctica didáctica } \\
3.2 .4,3.2 .5,3.2 .6\end{array}$ \\
\hline $\begin{array}{l}\text { Nivel simbólico: } \\
13 \equiv \eta 3\end{array}$ & $\begin{array}{l}\text { Deber-ser } \\
\text { Legisigno: forma del valor } \\
\text { Interpretación en el } \\
\text { sistema completo actual } \\
\text { de sus posibilidades } \\
\text { formales de AD para la } \\
\text { acción didáctica y para la } \\
\text { RD y la SD. } \\
\text { 1.3.7, 1.3.8, } 1.3 .9\end{array}$ & $\begin{array}{l}\text { Deber- hacer / actuar } \\
\text { Símbolo: valor de la existencia } \\
\text { interpretación y contextualización } \\
\text { en una situación de tiempo y } \\
\text { espacio determinados sobre la } \\
\text { gestión de una RD } \\
\text { 2.3.7, 2.3.8, } 2.3 .9\end{array}$ & $\begin{array}{l}\text { (9) deber-saber/compromiso } \\
\text { Argumento: valor del valor } \\
\text { interpretación a partir de un } \\
\text { determinado sistema de normas vigentes } \\
\text { sobre las prácticas didácticas, en la } \\
\text { evaluación y significación dela SD } \\
\text { 3.3.7, 3.3.8, 3.3.9 }\end{array}$ \\
\hline
\end{tabular}

Figura 5. Relación de planos, niveles y dinámicas de expresión de la competencia.

Fuente: elaboración propia

Por ejemplo, la figura 5 resume la aplicación de lo presentado en las configuraciones emergentes del proceso de sistematización y evaluación, así como los niveles de expresión de la competencia, los tipos de signos de los objetos meta-didácticos y su caracterización en el análisis semiótico del texto: en la lectura vertical, se relacionan tríadicamente a los tipos de prácticas (sentido); las caracterizaciones generales del objeto meta-didáctico correspondiente y los descriptores cualitativos de él -identificados con la codificación numérica, respectiva-. En la lectura horizontal, se identifican e indexan los niveles de expresión de la competencia y los tipos de signos en términos de los objetos didácticos en términos de los descriptores cualitativos de las competencias inferidos en esta investigación. 


\section{Discusión y presentación de los resultados de la evaluación de la competencia.}

La articulación a la semiótica peirceana que proponemos constructivamente de la caracterización de los planos, niveles y tipos de signos y tipos de descriptores cualitativos, es un aporte en la conceptualización de la acción didáctica. Puesto que ella, aplicada a la interpretación de las prácticas didácticas, permitirá un cierto grado de generalización en la validación del tipo de evaluación cualitativa por tendencias, rangos y rasgos de la competencia de los profesores en formación y en ejercicio de su profesionalidad.

\section{Semiótica de los textos en la evaluación de las competencias.}

Esta noción emergente de evaluación nos hace posible actualizar, utilizar y aplicar las nociones de los tipos de signos como herramienta y conceptualización en la exploración de los descriptores de los elementos de significado de los objetos didácticos y meta-didácticos. Evidentemente, también es posible aplicarla para el estudio los objetos didáctico-matemáticos y matemáticos escolares y creemos que se puede y se debe actualizar para otros campos disciplinares en el ámbito de lo educativo. En este trabajo aplicamos estas inferencias teórico-metodológicas de la siguiente manera:

- Planos de expresión semiótica de las competencias: Evaluamos la competencia al evaluar los planos de expresión de ella, en términos de identificar y caracterizar los elementos de significado del sentido de las prácticas didácticas, - existencia del subconjunto de las prácticas discursivas, operativas y normativas-,como primer filtro-criterio de tipo orientativo en la evaluación de la información.

- Niveles y tipos de signos de expresión de las competencias: Retomamos el concepto dinámico del signo asumido por Peirce, al considerarlo como la relación triádica que toma un aspecto percibido de algo (fundamento), en relación con ese algo (representamen) y lo presenta para alguien, reelaborado en un contexto específico a la consideración de ese alguien (interpretante). Esto permitió la ordenación de la información por niveles de expresión semiótica y de tipos de signos como indicadores de existencia de las competencias. La caracterización de estos tipos de signos y de los niveles de expresión semiótica dispone elementos que posteriormente serán aplicados en la identificación y caracterización de los descriptores semióticos de los objetos meta-didácticos que son objeto de investigación, - -síntomas de la expresión semiótica de las competencias-. Ello constituyó el segundo filtro-criterio evaluativo de tipo focal, pues posibilitó la identificación de los tipos de signos, en niveles de expresión semiótica que interpretamos como niveles icónico, indicial y simbólico.

- Tipos de descriptores cualitativos semióticos: Los descriptores fueron identificados ycaracterizados en los elementos de significado de los objetos meta-didácticos, se expresan en los textos en las unidades específicas de registro y se relacionan con los elementos de significado de la competencia $€(Q, P, C)$, en términos de la certificación de la presencia y valoración de los elementos de significado de los objetos meta-didácticos. De acuerdo con lo anterior, se garantizó la identificación y caracterización de dinámicas de expresión de la competencia, ello corresponde y está funcionalmente asociado al tipo de signo en una secuenciación en grados de expresión, como un tercer filtro, criterio de evaluación certificativo, validativo, regulativo. También posibilita la evaluación de la manifestación de los elementos de significado y la competencia en términos de sus descriptores modales querer/ poder/deber-ser/actuar/saber.

\section{Representación de la evaluación de las competencias.}

A los criterios adoptados se analiza y se hace una valoración semiótica cualitativa (no métrica) de la expresión de las competencias en los textos que son la presencia (extensión), la frecuencia (intensidad) y el orden (escalonamiento) de expresión de los códigos. La representación de la evaluación de las competencias, es emergente de la evaluación y representación de los planos, niveles y dinámicas presentados en los comentarios de los cuadernos 
y memorandos de registro, codificación y análisis (extensión); de las inferencias de las respuestas de orientación semiótica para la identificación y caracterización de los signos (intensidad); y de la identificación, valoración de los descriptores cualitativos emergentes en las tablas de análisis e interpretación de las unidades específicas de registro de la información.

Los números de identificación de las preguntas y respuestas están relacionados con los números asignados a los tipos de signos, posteriormente, ellos son utilizados en la representación de la evaluación de las competencias, como expresión de un "vector de la competencia". Es decir, que los análisis de los textos y su interpretación son como una ruta de expresión semiótica de la competencia y su representación gráfica del "vector de la competencia". Estos son indicadores de la expresión de las competencias, para después caracterizar con ellos los descriptores semióticos en términos de los descriptores cualitativos de los objetos meta-didácticos, - dinámicas de configuración de la competencia - como criterio de su evaluación (figura 6).

- Ejemplo de evaluación de la competencia en las prácticas discursivas: La presencia, frecuencia y orden de los códigos identificados y evaluados en las unidades específicas de análisis, contrastada tríadicamente con las evidencias en las unidades focales de análisis y con la información dispuesta en las unidades generales de análisis permite inferir que: en las prácticas discursivas se evidencia claramente la existencia de la competencia para el análisis didáctico con intermediación de íconos como signos más representativos que están asociados a la reflexión discursiva (RD) y al sentido valorativo (SD) en un nivel icónico primordialmente, querer/poder/saber-ser.

Es decir, que los análisis de los textos y su interpretación como una ruta de expresión de la competencia y su representación gráfica del "vector de la competencia" para las prácticas discursivas muestran, en la figura 6 , la trayectoria vectorial de las competencias expresadas en las textualizaciones de las prácticas discursivas así:
1) 1-2-3: una fuerte presencia del querer-ser desde el primer nivel del análisis didáctico - percepción de los elementos de significado didáctico-matemático-asociados al querer-actuar, primer nivel de la reflexión didáctica (gramático-descriptiva) y a la valoración del querer-comprometerse, primer nivel de la semiosis didáctica (material-retórica).

2) 7-2-3: una muy significativa presencia desde el tercer nivel del análisis didáctico del deber-ser (configuraciones didáctico-matemáticas), asociadaal querer-actuar, primer nivel de la reflexión didáctica (gramático-descriptiva) y a la valoración del querer-comprometerse, primer nivel de la semiosis didáctica (material-retórica).

3) 4-2-3: de manera puntual, marginal y débil, la presencia desde el segundo nivel del análisis didáctico del poder-ser (tipos de significado), asociado al querer-actuar, primer nivel de la reflexión didáctica (gramático-descriptiva) y a la valoración del querer-comprometerse, primer nivel de la semiosis didáctica (material-retórica).

\begin{tabular}{|c|c|c|c|}
\hline $\begin{array}{l}\text { Práctica } \\
\text { discursiva }\end{array}$ & $\begin{array}{l}\text { Ser } \\
\text { AD }\end{array}$ & $\begin{array}{c}\text { Actuar-hacer } \\
\text { RD }\end{array}$ & $\begin{array}{c}\text { Saber-conocer } \\
\text { SD } \\
\end{array}$ \\
\hline $\begin{array}{c}\text { Querer } \\
\eta_{1}\end{array}$ & $\begin{array}{c}1 . \\
1.1 .1 \\
1.1 .2 \\
11.3\end{array}$ & $\begin{array}{c}2 . \\
2.1 .1 \\
2.1 .2 \\
4.1 .3\end{array}$ & $\begin{array}{l}3 . \\
3.1 .1 \\
3.1 .2 \\
3.1 .3\end{array}$ \\
\hline $\begin{array}{c}\text { Poder } \\
\eta_{2}\end{array}$ & $\begin{array}{c}4 . \\
1.2 .4 \\
1.2 .5 \\
1.2 .6\end{array}$ & $\begin{array}{c}5 . \\
2.2 .4 \\
2.2 .5 \\
2.2 .6\end{array}$ & $\begin{array}{c}6 . \\
3.2 .4 \\
3.2 .5 \\
3.2 .6\end{array}$ \\
\hline $\begin{array}{c}\text { Deber } \\
\text { Compromiso } \\
\eta_{3}\end{array}$ & $\begin{array}{c}7 . \\
1.3 .7 \\
1.3 .8 \\
1.3 .9\end{array}$ & $\begin{array}{c}8 . \\
2.3 .7 \\
2.3 .8 \\
2.3 .9\end{array}$ & $\begin{array}{c}9 . \\
3.3 .7 \\
3.3 .8 \\
3.3 .9\end{array}$ \\
\hline
\end{tabular}

Figura 6. Evaluación de la competencia de análisis didáctico, prácticas discursivas.

Fuente: elaboración propia

El orden de regularidad semiótica de los descriptores cualitativos de la competencia para el análisis didáctico asociadoa los descriptores cualitativos de la reflexión didáctica y de la semiosis didáctica, que inferimos en las prácticas discursivas textualizadas del EPM es: 
1. Querer-ser/actuar/saber (1-2-3): Percepción del discurso de elementos de significado didáctico, relacionado con su intelección para la acción reflexiva y asociados la identificación de la validez de la posible práctica didáctica.

2. Deber-ser/actuar/saber (7-2-3): Valoración de la imaginación y conocimiento de los elementos de la relación profesor-estudiante, relacionado con su intelección para la acción reflexivay asociados laidentificaciónde la validez de la posible práctica didáctica.

3. Poder-ser/actuar/saber (4-2-3): Sensibilización $y$ consideración de los tipos de significado, relacionado con su intelección para la acción reflexivay asociados la identificación de la validez de la posible práctica didáctica.

Ello lo interpretamos y valoramos como: alto grado de competencia para la descriptibidad de la práctica didáctica, con grado medio de nivel de valoración explicita del sentido de la práctica didáctica y grado bajo de nivel reflexividad sobre esa descripción de la posible práctica didáctica. Nuestra inferencia valorativa es de un alto nivel de idoneidad discursiva en AD siempre asociado a un nivel icónico de RD y SD - expresiones de la forma de la práctica didáctica asociada a nivel de formalidad de la RD y a un nivel de la forma del valor SD-.

Este procedimiento de caracterización y representación del proceso evaluativo se realizó para los tipos de prácticas operativas y normativas. La figura 7 muestra nuestra abducción evaluativa de las competencias en el conjunto de una identificación específica de los descriptores cualitativos correspondientes a la competencia $€(\mathrm{Q}, \mathrm{P}, \mathrm{C} \Xi$ [querer/poder/deber-ser/actuar/saber]), en relación con los elementos de significado de los objetos meta-didácticos $(\mathrm{AD}, \mathrm{RD}, \mathrm{SD})$.

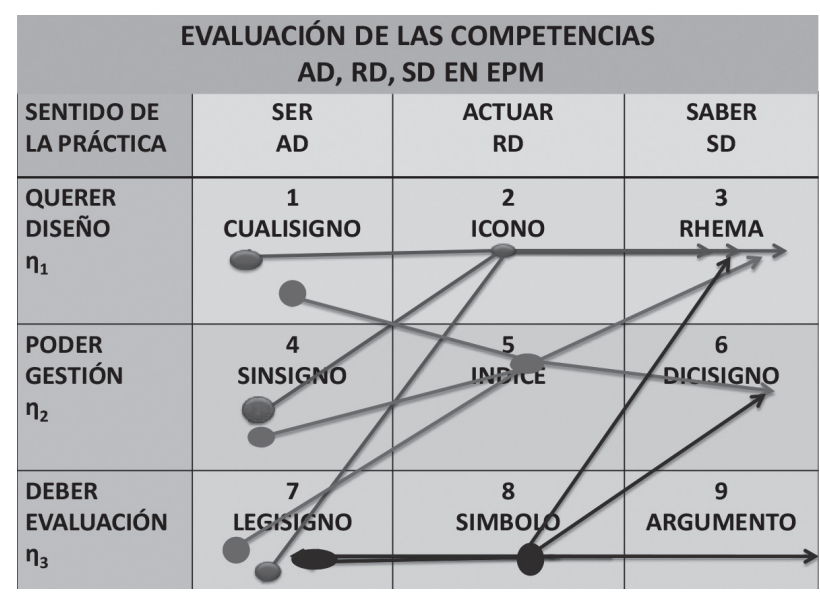

Figura 7. Representación general de la evaluación de las competencias.

Fuente: elaboración propia

Asumimos la interpretación-inferencia abductiva de los tipos de descriptores cualitativos, como elementos primarios de $€(\mathrm{Q}, \mathrm{P}, \mathrm{C})$, como expresión de los elementos de identificación y descripción de las competencias, - secuenciación de grados de expresión de la competencia y se infieren los elementos cualitativos primarios de evaluación certificativa de las competencias-.

\section{Los tipos de descriptores cualitativos de expresión de las competencias.}

En todos los casos, los tipos de descriptores cualitativos se identifican como textualización de los elementos de significado de los objetos-procesos meta-didácticos ( $\mathrm{AD}, \mathrm{RD}, \mathrm{SD})$, en términos de los elementos de significado de las competencias (querer-ser/actuar/saber), (poder-ser/actuar/ saber), (deber-ser/actuar/saber), (ver figura 3 y 7 ).

Se pueden identificar en los tres niveles (icónico, indicial, simbólico), en relación con los tipos de signos, que se expresan como indicadores de las competencias, por lo tanto son elementos de certificación y evaluación cualitativa de ellas. Ellos evidencian la dinámica de los procesos de significaciónen los procesos de estudio didáctico y meta-didáctico asociados a la sensibilidad individual (querer-ser/actuar/saber); identidad colectiva con los comportamientos y acciones de un EPM (poder-ser/actuar/saber); 
autoafirmación profesional de los EPM (deberser/actuar/saber) como valoración de sus actividades como docente.

Estos descriptores cualitativos hacen referencia y están asociados a los procesos de valoración de la abducción y síntesis de la de implicación con teorías praxeológicas de tipo didáctico-matemáticas en la práctica didáctica y que representan niveles de idoneidad didáctica por parte del EPM.

1. Percepción del discurso de elementos de significado didáctico, discursividadpro-activa para la acción, sensibilidadvalorativapara los resultados de la posible práctica didáctica.

2. Intelección para la acción reflexiva; decisión en la interacción didáctica; regulación y verificación en la reflexión sobre la acción didáctica.

3. Identificación de la validez de la interpretación; pertinencia y coherencia en las posibles valoraciones; confianza y solidaridad en el sentido de sus significaciones de tipo didáctico.

4. Sensibilización y consideraciónde los tipos de significado; afectación y ajuste reflexiva en la acción didáctica; valoración y gusto por la actividad reflexiva en la práctica didáctica.

5. Evocación intelectiva de objetivos de la práctica didáctica; interpretación y formalidad en la formulación de soluciones; consenso y validaciónde la gestión y de la actividad reflexiva en la práctica didáctica.

6. Identificación de la validez de la gestión didáctica; solidaridad en la reflexión sobre la acción; pertinencia y coherencia en las posibles valoraciones de la actividad didáctica; confianza en el sentido de sus significaciones pedagógicas en la práctica didáctica.

7. Valoración de la imaginación y conocimiento de los elementos de la relación profesor-estudiante; creatividad y disfrute en la acción didáctica; gusto y motivación de que hace uso para la práctica didáctica.
Comprensión, consenso, revisión y aplicación de las normas y hábitos de comportamiento; interpretación, representación y valoraciónde las estrategias de uso didáctico; productividad, renovación e implicación con teorías y praxeologías de tipo didáctico.

Justificación y argumentación de las aserciones; la relación o correspondencia entre los significados; pertinencia y cambio en las decisionesdidácticas para la solución de problemas didácticos; compromiso pedagógico y didáctico institucional.

Los descriptores cualitativos son nuestras caracterizaciones inferidas en los textos de las percepciones, consideraciones y motivaciones discursivas de la práctica didáctica. Son deducidos de nuestra interpretación con relación a las actualizaciones de acciones, reacciones o certificaciones en la práctica didáctica (ver gráficas 3, 4, 5 y 7), por ejemplo, se pueden inferir:

- Como expresiones del querer-ser (AD), expresiones del querer-actuar (RD) y expresiones del querer-saber (SD), en el nivel icónico de expresión de las competencias y sus correspondientes tipos de signos.

- Como expresiones del poder-ser (AD), poder-actuar (RD) y poder-saber (SD), en el nivel indicial de expresión de la competencia y sus respectivos tipos de signos.

- Como expresiones del deber-ser (AD), deber actuar (RD) y deber saber (SD) en el nivel simbólico de expresión de la competencia y sus correspondientes tipos de signos.

\section{Evaluación de la competencia para... y la idoneidad didáctica en ep.}

Los ideales de conducta a los que se refería Peirce, que nosotros llamamos en este trabajo idoneidad didáctica, pueden ser evaluados en el campo de la formación de profesores de matemáticas, en el caso de las prácticas didácticas de los EPM y teniendo como implicación pragmática la evaluación de las competencias.

Por ejemplo, los descriptores cualitativos son nuestra abducción de la valoración efectiva expresada 
en los textos como normas, hábitos y reglas sobre la práctica didáctica. En tanto que se pueden inferir como el deber-ser (AD), deber-actuar (RD) y eldeber-saber (SD), en el nivel simbólico de expresión de la competencia y sus respectivos tipos de signos. Ellos se expresan en tipos de prácticas discursivas operativas y normativas, que nos permitieron configurar y graficar la trayectoria de la evaluación de la competencia - vector de la competencia-, es decir,los números asignados a los tipos de signos que posteriormente son utilizados en la representación de la evaluación de las competencias, (ver figuras 6 y 7).
Como lo presenta y resume la figura 8, en una lectura vertical de los "ideales de conducta" los componentes de los elementos de significado de los objetos meta-didácticos, resumidos en las expresiones de la figura, se componen de manera operativa y funcional como objetos que expresan la competencia, también de manera recursiva se comportan como criterios y filtros epistemológicos para su evaluación, y de manera hologramática se institucionalizan como representaciones simbólicas de los mismos elementos de significado de la competencia (querer/ poder/saber-ser/actuar/deber) que se manifiestan y expresan en los textos de los EPM.

\begin{tabular}{|c|c|c|c|c|}
\hline & ica & $\begin{array}{l}\text { FORMA } \\
\text { LA PRÁCTICA DIDÁctICA } \\
\text { Análisis didáctico } \\
\text { SER }\end{array}$ & $\begin{array}{c}\text { EXISTENCIA } \\
\text { LAPRÁCTICA DIDÁCTICA } \\
\text { Reflexión didáctica } \\
\text { ACTUAR }\end{array}$ & $\begin{array}{c}\text { VALOR } \\
\text { LA PRÁCTICA DIDÁCTICA } \\
\text { Semiosis didáctica } \\
\text { SABER }\end{array}$ \\
\hline $\mathbf{Q}$ & $\begin{array}{l}\text { Diseñoy } \\
\text { planeación } \\
\text { Posibilidad de } \\
\text { una práctica } \\
\text { didáctica }\end{array}$ & $\begin{array}{l}\text { 1. Repertorio de las } \\
\text { formas de modelar } \\
\text { la práctica } \\
\text { didáctica: } \\
\text { CUALISIGNO }\end{array}$ & $\begin{array}{l}\text { 2. Actualización de } \\
\text { situaciones y } \\
\text { agendas para una } \\
\text { práctica didáctica: } \\
\text { ÍcONO }\end{array}$ & $\begin{array}{l}\text { 3. Armonía y } \\
\text { congruencia de } \\
\text { Valoración de la en la } \\
\text { práctica didáctica: } \\
\text { RHEMA }\end{array}$ \\
\hline $\mathbf{P}$ & $\begin{array}{l}\text { Gestión } \\
\text { Como } \\
\text { actualización de } \\
\text { una práctica } \\
\text { didáctica }\end{array}$ & $\begin{array}{l}\text { 4. Repertorio concreto } \\
\text { de recursos } \\
\text { disponibles. } \\
\text { Propiedades en una } \\
\text { situación, sociedad y } \\
\text { tiempo: } \\
\text { SINSIGNO }\end{array}$ & $\begin{array}{l}\text { 5. Rutina cotidiana } \\
\text { Puesta en acto de la } \\
\text { agenda didáctica. } \\
\text { Repetición } \\
\text { automatismo } \\
\text { Procedimientos: } \\
\text { ÍNDICE }\end{array}$ & $\begin{array}{l}\text { 6. Confrontación } \\
\text { de la rutina diaria en } \\
\text { el mundo exterior. } \\
\text { Rendimiento } \\
\text { productividad y } \\
\text { valor: } \\
\text { DECISIGNO }\end{array}$ \\
\hline & $\begin{array}{c}\text { Evaluación y } \\
\text { normación } \\
\text { Como } \\
\text { compromiso } \\
\text { con la práctica } \\
\text { didáctica }\end{array}$ & $\begin{array}{l}\text { 7. Hábitos y rutinas } \\
\text { de regulaciones } \\
\text { didácticas y } \\
\text { pedagógicas de una } \\
\text { práctica didáctica: } \\
\text { LEGISIGNO }\end{array}$ & $\begin{array}{l}\text { 8. Regulación y roles } \\
\text { didáctica de las } \\
\text { normas para la } \\
\text { eficiencia de una } \\
\text { práctica didáctica: } \\
\text { SIMBOLO }\end{array}$ & $\begin{array}{l}\text { 9. Aprobación social, } \\
\text { valoración cualitativa } \\
\text { pedagógica para la } \\
\text { valoración de la } \\
\text { práctica didáctica: } \\
\text { ARGUMENTO }\end{array}$ \\
\hline
\end{tabular}

Figura 8. Generalización semiótica de la evaluación de las competencias.

Fuente: elaboración propia

También en una lectura horizontal se resumen los "ideales de conducta" para los objetos didácticos. En la lectura horizontal los niveles y tipos de expresión de los elementos de significado de la competencia querer/poder/deber-ser/actuar/saber, en las intersecciones triádicas las respectivas generalizaciones de los descriptores cualitativos de la competencias. Igualmente, dicha configuración dispone el proceso de generalización asumido por nosotros para la evaluación de las competencias propuesta en esta investigación, pues en ella se representaron cualitativamente los resultados de dicha evaluación, una vez fueron actualizados los procesos de interpretación-análisis de la información. En este caso, podríamos aplicarle a esta figura la representación "vector de la competencia", hacer juicios de idoneidad y generalizar como evaluación para LEBEM sobre sus intencionalidades de formación, para una institución escolar, o para una práctica social en un campo de las interacciones humanas. 


\section{Conclusiones, aportes y perspectivas}

En este trabajo, desde el rastreo y revisión bibliográfica, se mostró que, internacional y nacionalmente, en los últimos años se ha propuesto y desarrollado la política de gestión de la calidad educativa que, focalmente, una de sus principales realizaciones ha sido la implementación del enfoque de competencias en todos los niveles educativos, como específicamente se evidenció con la manera estratégica, preventiva y regulativa como se ha utilizado este enfoque para diseñar, gestionar y evaluar dicha política educativa. También se mostró que, así como se ha especificado y puesto un gran énfasis a la temática de la evaluación de las competencias desarrollas en los diferentes niveles de los sistemas educativos, también se han puesto en evidencia las tensiones, problemas, necesidades de investigación, sustentación, estudio y evaluación, tanto de la mencionada política, como su actualización educativa mediante el enfoque de competencias.

\section{La investigación en la formación de profesores de matemáticas.}

En el campo de la Educación Matemática, según Lurduy (2010), los principales investigadores, plantean la necesidad de prestar atención a esta temática y proponen enfocar los estudios a la investigación en formación de profesores de matemáticas. De manera general y como un primer resultado de esta investigación, se puede inferir que los desarrollos investigativos sobre la formación de profesores de matemáticas, apuntan a la investigación estudio y evaluación de los programas de formación de profesores de matemáticas, a los aspectos relacionados con el conocimiento profesional de los profesores, a la evaluación de la idoneidad profesional de los profesores y de los estudiantes para profesor de matemáticas (EPM).

En este estudio fueron confirmados e identificados problemas y temáticas prioritarias de investigación, como el conocimiento profesional del profesor, los procesos de diseño, gestión y evaluación de prácticas matemáticas en el aula, los aspectos pedagógicos y didácticos generales asociados a las acciones didácticas, los aspectos de desarrollo profesional del profesor de matemáticas relacionados con la idoneidad didáctica. Además de estos temas y en relación con ellos, se evidenció que uno de las más plausibles soluciones a las problemáticas de la falta de recursos teórico-metodológicos, en este campo de investigación, es la pertinencia, confiabilidad y rigor de la aplicación de la semiótica como método para abordar las problemáticas del estudio de las entidades matemáticas y didácticas, así como para la evaluación de las competencias.

En esta dirección, esta investigación logró adaptar, aplicar e implementar los desarrollos teórico-metodológicos del EOS y la perspectiva semiótica peirceana, desarrollados para las prácticas matemáticas en el aula y utilizarlos en los proceso de evaluación de la idoneidad didáctica de los EPM, en el sentido de proponer, aplicar y utilizar instrumentos para evaluar las competencias de análisis, reflexión y semiosis didáctica. En este sentido, la investigación que presentamos en este artículo, esun estudio evaluativo, que aporta algunos aspectos, recursos, herramientas e instrumentos de tipo teórico-metodológico a la apuestas formativas de profesores de matemáticas, como por ejemplo al programa de Licenciatura en Educación Básica con Énfasis En Matemáticas, (LEBEM).

\section{Problema, preguntas y objetivos de investigación.}

Delimitamos nuestra acción investigativa de tipo evaluativo a algunas de las producciones escritas de los EPM, que había sido el resultado de los procesos de estudio didáctico en los espacios formativos de la práctica docente, en dicho programa de formación de profesores de matemáticas. De acuerdo con lo anterior, hicimos proyectables y configurables esos recursos, herramientas e instrumentos de tipo semiótico en lo teórico-metodológico a la evaluación de las competencias en otros niveles educativos e incluso para otros objetos de estudio en el campo educativo o en la indagación de la generalidad de los fenómenos sociales.

En particular, hemos logrado especificar los resultados de investigación a la evaluación de las competencias de análisis, reflexión y semiosis didáctica expresadas en dichos textos de los EPM, cuando ellos diseñan, 
gestionan y evalúan una práctica didáctica en el aula, para un proceso de estudio matemático escolar.

\section{Conceptualización de las competencias y de un método para su evaluación.}

En los referentes teóricos y metodológicos de esta investigación, hicimos una apuesta teórica (compleja semiótica y transcultural), que nos permitió hacer una caracterización de las competencias $(€(\mathrm{Q}, \mathrm{P}, \mathrm{C} \Xi$ [querer/poder/deber-ser/actuar/ saber]), y respectivamente implementamos instrumentos para evaluar la competencia para el análisis, reflexión y semiosis didáctica de los profesores de matemáticas en formación.

Mediante un proceso de articulación, combinación y asociación metodológica entre diferentes principios, estrategias y técnicas de TFD-ACC-AST, desarrollamos un procedimiento para evaluar las competencias para el análisis, reflexión y semiosis didáctica de los EPM, cuando ellos diseñan, gestionan y evalúan una práctica matemática escolar, yuna práctica-didáctica. Finalmente, lo anterior aporta elementos que pueden configurar y proyectar elementos de significado de tipo didáctico y meta-didáctico que evidencian algunos procedimientos para el estudio e investigación de la formación didáctico-matemática de los EPM.

Partiendo de los desarrollos e indagaciones logradas en TFD-ACC, se posibilitó, por un lado, reducir y determinar los casos y los textos estudiados, y por otro lado, identificar los tipos de textos, y cómo ellos evidenciaban la presencia de los elementos de las competencias, así como identificar los tipos de prácticas significativas expresadas en los textos. Igualmente, por los procedimientos implementados en ACC-AST, se identificaron y caracterizaron los elementos de significado de los objetos didácticos y meta-didácticos que contribuyen a configurar los elementos teórico-metodológicos, para la evaluación de dichas competencias.

En ACC-AST identificamos la existencia y la presencia del signo de la competencia de un EPM:

Considerada la estructura del signo como objeto, representamen, interpretante;la primera parte de cada una de tales denominaciones corresponde a la cualidad-forma-consideración que debe tener el fundamento del signo; la segunda a la existencia-acción-interpretación del representamen del signo; y la tercera a la valoración-significación-normación del interpretante del signo. En ACC-AST evaluamos el tipo de significado de un objeto didáctico asociado a algún elemento de significado del análisis y reflexión didáctica, identificando lo relativo al aspecto característico de la competencia (querer-ser, poder-ser, deber-ser) y, a partir de ello, en AST-TFD, evaluamos el tipo designo que evidencia un tipo de práctica (discursiva, operativa normativa) asociado al rasgo del aspecto característico de la competencia (querer-ser, poder-ser, deber-ser).

Con esta forma triádica de evaluar la expresión de los elementos de significado de las competencias de un EPM: en AST-TFD pudo obtenerse un conjunto trayectorias que representan las formas, las acciones y las valoraciones de la práctica didáctica del EPM. Interpretada la estructura del signo como objeto (sentido, $\mathrm{PD}, \mathrm{PO}, \mathrm{PN})$, representamen $(\mathrm{Q}, \mathrm{P}$, $\mathrm{C}$ expresadas en niveles y tipos de signos), interpretante (descriptores cualitativos de AD, RD, SD).

Nuestras evidencias de la complejidad de la situación didáctica en la que intervienenlos sujetos-actores apuntan a la hipótesis de que el análisis puede ser reflexivo, pero no necesariamente presentarse en y sobre la acción (práctica). En este sentido, es que la reflexión didáctica a la que nos referimos es primariamente analítica sobre la acción didáctica -también previa a la acción-, pero no sólo prescriptiva y por tanto es posible caracterizarla como diferente del análisis didáctico.

Todo el proceso desarrollado en TFD-AST permite evidenciar respuestas a las preguntas específicas sobre las características de la competencia para el análisis, reflexión y semiosis didáctica en estudiantes para ser profesor de matemáticas, al identificar descriptores cualitativos, indicadores y niveles de expresión semiótica de las competencias identificados en los tipos de prácticas textualizadas por los EPM.

Mediante la utilización de la semiótica como metodología en ACC-AST y en AST-TFD, para la caracterización e identificación del significado y el 
sentido de las prácticas didácticas textualizadas se determinóel procedimiento para que pudieran ser evaluados aspectos específicos de las competencias para el análisis y la reflexión didáctica en relación a los profesores en formación (EPM), y a la práctica docente de los EPM.

\section{Aportes y prospectivas de la investigación}

Partimos de las apuestas teórico-metodológicas de EOS para las prácticas matemáticas,e hicimos nuestra interpretación y adaptación de ellas para el caso de las prácticas didácticas y con la contribución de consideraciones de tipo filosófico-educativo, pedagógico-didáctico, en educación matemática, en didáctica de las matemáticas, en la formación de profesores de matemáticas, reconstruimosel significado de la noción de competencia y evidenciamos procedimientos para su evaluación enel caso de los EPM, con las siguientes conclusiones y aportes que se convierten en nuevos camposde exploración, investigación y estudio:

1) Identificamos las formas, organizaciones y significados de las prácticas didácticas, expresadas en los textos de los EPM de LEBEM, para la adecuada e idónea caracterización de los objetos didácticos y meta-didácticos para la evaluación de las competencias en el análisis, reflexión y semiosis didáctica.

2) Caracterizamos y conceptualizamos los elementos de significado de los objetos didácticos y meta-didácticos expresados en las unidades de análisis de los textos de los EPM, que posibiliten la evaluación de la competencia para el análisis, reflexión y semiosis didáctica desarrolladas en su práctica docente.

3) Identificamos el significado y el sentido de las prácticas didácticas presentes en las unidades generales de análisis en los textos de los EPM, que evidencian la existencia y expresión de los elementos característicos de la competencias y actualizan su evaluación.

4) Establecimos niveles e indicadores de expresión semiótica de los elementos característicos y las competencias en las unidades específicas de análisis en los textos, para la valoración cualitativa, las competencias de análisis, reflexión y semiosis didáctica.
5) Determinamos y evaluamos en las unidades específicas de análisis los descriptores cualitativos de expresión semiótica de la competencia para el análisis, reflexión y semiosis didáctica de los EPM.

6) Sistematizamos y proponemos un proceso de evaluación los elementos característicos de la competencia para el análisis reflexión y semiosis didáctica de los EPM.

7) Evaluamos los elementos de significado de la noción de la competencia, que contribuyan a configurar los elementos teórico-metodológicos, para la evaluación de dicha competencia.

8) Aportamos herramientas teóricas y metodológicas a los programas de formación de profesores, formadores de profesores y a los profesores de matemáticas en la evaluación de la competencia para el análisis, reflexión y semiosis didáctica.

9) Mostramos que es posible diseñar, gestionar y evaluar un proceso de evaluación de los elementos de significado de la competencia que posibiliten delinear, implementar y valorar la idoneidad didáctica de estudiantes en formación para profesor, de profesores y de formadores de profesores de matemáticas.

10) La inducción-abductiva en el análisis de los tipos de prácticas textualizadas; la deducción-abducción de las expresiones textuales de niveles y tipos de signos de expresión de la competencias; la síntesis-abductiva de los descriptores cualitativos de expresión de los elementos de significado de las competencias, son adaptados, aplicados y significados como la representación vectorial de expresión de la competencia, para evaluar las expresiones de los elementos de significado de ellas (querer/poder/saber-ser/actuar/deber), lo anterior podráser investigado en el caso de las matemáticas escolares en los procesos de estudio y en las prácticas matemáticas en el aula.

11) La utilidad de esta síntesis-abductiva en términos de descriptores de los elementos de significado de la competencia se puede apreciar interpretando los resultados de la extensividad, intensividad y orden de expresión semiótica — según ese orden-, identificados en las tablas del análisis 
de la información - tipos de prácticas textualizadas-, ellos plantean nuevos aspectos y desarrollos que deben y pueden ser investigados en el campo de la educación matemática y en aspectos específicos de la formación de profesores.

12) Nuestra interpretación del esquema peirceano muestra los resultados del análisis en una representación vectorial de la trayectoria de la competencia en términos de los objetos meta-didácticos y sus descriptores y abre un nuevo campo de investigación.

Por lo anterior, creemos que hemos aportado herramientas e instrumentos para evaluar la idoneidad didáctica de un profesor o de un profesor de matemáticas y hemos desarrollado teórico-metodológicos para la evaluación en los diferentes niveles educativos de los desarrollos de la gestión y mejora de la calidad educativa.

En particular y de manera específica, desde los desarrollos investigativos de EOS y nuestra interpretación y adaptación de la semiótica peirceana, evidenciamos que se pueden aportar elementos de significado didáctico y meta-didácticos para una mejor aplicación, comprensión y significación de la formación de profesores de matemáticas.

Desde una perspectiva de formación y desde una manera de afrontar las prácticas matemáticas escolares, los formadores de niños y jóvenes pueden, evaluar y mejorar sus propias prácticas, aplicando las herramientas semióticas desarrolladas en esta investigación. Ello es así ya que sus propias acciones y las de sus estudiantes se expresan en escritos, exposiciones, videos, carteles, cuadernos, documentos, y otros, en definitiva, material textual cuyo análisis, reflexión y semiosis necesita aplicar herramientas conceptuales y metodológicas como las desarrolladas en esta investigación, convenientemente adaptadas a las prácticas matemáticas en el aula.

\section{Referencias}

Abril, G. (1994). Análisis semiótico del discurso. En: Delgado, J, M. y Gutiérrez, J. (Eds.). Métodos y técnicas cualitativas de investigación en ciencias sociales. Madrid: Síntesis.
Bardin, L. (1986). Análisis de contenido. Madrid: Akal/Universitaria.

Godino, J. D. y Cols. (1991-2012). Teoría de la Educación Matemática. Universidad de Granada: Grupo de investigación. Teoría y metodología de investigación en Educación Matemática. Disponible en: http://www.ugr.es/local/jgodino

Lurduy, O. (2010). Investigación en la formación de profesores de matemáticas. Agendas y perspectivas.Revista científica. (11). Bogotá: Universidad Distrital.

Lurduy, O. (2009). "El profesor investigador de su práctica. En La Formación Del Profesorado De Matemáticas". Uno: Revista de didáctica de las matemáticas. (51). Barcelona: Grao.

Lurduy, O. (2013). Conceptualización y evaluación de las competencias para el análisis reflexión $y$ semiosis didáctica. El caso de los estudiantes para profesor de matemáticas. (Tesis doctoral inédita). Bogotá: Universidad Distrital. DIE-UD.

Morín. E.(1988). El método 3. El conocimiento del conocimiento. (Trad. de Ana Sánchez y Dora Sánchez). Madrid: Editorial Cátedra.

Niño, D. (2007). Emergencia de la Semeiótica Universal en C. S. Peirce. (Diapositivas guiadas). Bogotá: Seminario CSP- Universidad Nacional de Colombia.

Navarro, P. y Díaz, C. (1995). Análisis de contenido. En: J.M. Delgado y J. Gutiérrez. (Coord.). Métodos y Técnicas cualitativas de investigación en Ciencias Sociales. Madrid: Síntesis.

Peirce, C. S. (1971). Cómo hacer claras nuestras ideas. En: Mi alegato a favor del pragmatismo. Buenos Aires: Editorial Aguilar.

Strauss, L. y Corbin, M. (2002). Bases de la investigación cualitativa. Técnicas y procedimientos para desarrollar la teoría fundamentada. Medellín: Editorial Universidad de Antioquia.

Vasilachis, I. (2006). Estrategias de investigación cualitativa. Barcelona: Editorial Gedisa. 